\section{A pore scale analysis for determination of interfacial convective heat transfer coefficient for thin periodic porous media under mixed convection}

Hasan Celik

Department of Mechanical Engineering, Izmir Institute of Technology, Urla, Turkey

Moghtada Mobedi

Faculty of Engineering, Shizuoka University, Hamamatsu, Japan

Oronzio Manca

Dipartimento di Ingegneria Industriale e dell'Informazione, Seconda Universita degli Studi di Napoli, Aversa, Italy, and

Unver Ozkol

Izmir Institute of Technology, Department of Mechanical Engineering, Izmir, Turkey

\begin{abstract}
Purpose - The purpose of this study is to determine interfacial convective heat transfer coefficient numerically, for a porous media consisting of square blocks in inline arrangement under mixed convection heat transfer.

Design/methodology/approach - The continuity, momentum and energy equations are solved in dimensionless form for a representative elementary volume of porous media, numerically. The velocity and temperature fields for different values of porosity, Ri and Re numbers are obtained. The study is performed for the range of Ri number from 0.01 to 10 , Re number from 100 to 500 and porosity value from 0.51 to 0.96 . Based on the obtained results, the value of the interfacial convective heat transfer coefficient is calculated by using volume average method.

Findings - It was found that at low porosities (such as 0.51), the interfacial Nusselt number does not considerably change with Ri and Re numbers. However, for porous media with high Ri number and porosity (such as 10 and 0.51 , respectively), secondary flows occur in the middle of the channel between rods improving heat transfer between solid and fluid, considerably. It is shown that the available correlations of interfacial heat transfer coefficient suggested for forced convection can be used for mixed convection for the porous media with low porosity (such as 0.51 ) or for the flow with low Ri number (such as 0.01 ).

Originality/value - To the best of the authors' knowledge, there is no study on determination of interfacial convective heat transfer coefficient for mixed convection in porous media in literature. The present study might be the first study providing an accurate idea on the range of this important parameter, which will be useful particularly for researchers who study on mixed convection heat transfer in porous media, macroscopically.
\end{abstract}

Keywords Porous media, Interfacial heat transfer coefficient, Mixed convection heat transfer

Paper type Research paper

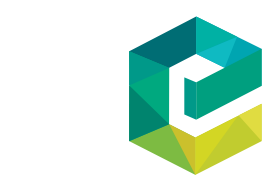

International Journal of Numerical Methods for Heat \& Fluid Flow Vol. 27 No. 12,2017 pp. $2775-2798$

c) Emerald Publishing Limited 0961-5539 DOI 10.1108/HFF-01-2017-0036 


\section{Nomenclature}

27,12

$\mathrm{A}=$ Area $\left[\mathrm{m}^{2}\right]$;

$A_{s f}=$ Interfacial area between solid and fluid phases $\left[\mathrm{m}^{2}\right]$;

$A_{s s}=$ Specific solid - fluid interface area $\left[\mathrm{m}^{2} / \mathrm{m}^{3}\right]$;

$\mathrm{A}^{*}=$ Aspect ratio [2L/was shown in Figure 2];

$\mathrm{D}=$ Dimension of the solid particle in both $\mathrm{x}$ and $\mathrm{y}$ directions;

$\mathrm{g}=$ Gravitational acceleration $\left[\mathrm{m} / \mathrm{s}^{2}\right]$;

Gr = Grashof number, equation (5);

$\mathrm{H}$ = Distance between the center points of each square blocks;

hint $=$ Local interfacial convective heat transfer coefficient $\left[\mathrm{W} / \mathrm{m}^{2} \mathrm{~K}\right]$;

$\mathrm{k}=$ Thermal conductivity $[\mathrm{W} / \mathrm{mK}]$;

$\mathrm{L} \quad=$ Length $[\mathrm{m}]$;

$\overline{\mathrm{Nu}}=$ Average Interfacial Nusselt number;

$\mathrm{Nu}_{\mathrm{L}}=$ Local Interfacial Nusselt number;

$\mathrm{Nu}_{\mathrm{Lc}}=$ Contribution to Local Nusselt number;

$\mathrm{p} \quad=$ Pressure [Pa];

$\mathrm{P} \quad=$ Dimensionless pressure, equation (5);

$\operatorname{Pr} \quad=$ Prandtl number, $\nu / \alpha ;$

Re = Reynolds number, equation (5);

Ri = Richardson number, equation (5);

$\mathrm{T}=$ Temperature [K];

$\vec{u}=$ Velocity vector;

$\mathrm{u} \quad=$ Velocity in $\mathrm{x}$-direction $[\mathrm{m} / \mathrm{s}]$;

$\mathrm{v} \quad=$ Velocity in $\mathrm{y}$-direction $[\mathrm{m} / \mathrm{s}]$;

$\mathrm{u}^{*}=$ Dimensionless velocity in $\mathrm{x}$-direction;

$\mathrm{v}^{*}=$ Dimensionless velocity in $\mathrm{y}$-direction;

$\mathrm{V}=$ Volume $\left[\mathrm{m}^{3}\right]$;

$\mathrm{w}=$ Width $[\mathrm{m}]$;

$\mathrm{x}, \mathrm{y}=$ Dimensional axial and transverse directions [m]; and

$\mathrm{X}, \mathrm{Y}=$ Dimensionless axial and transverse directions.

\section{Greek symbols}

$\alpha=$ Thermal diffusivity of the fluid $\left[\mathrm{m}^{2} / \mathrm{s}\right]$;

$\beta=$ Thermal expansion coefficient $[1 / \mathrm{K}]$;

$\varepsilon=$ Porosity;

$\theta=$ Dimensionless temperature, equation (5);

$\mu=$ Dynamic viscosity [Pas]; and

$\nu=$ Kinematic viscosity $\left[\mathrm{m}^{2} / \mathrm{s}\right]$.

\section{Subscripts and superscripts}

$$
\begin{aligned}
& \mathrm{f} \quad \text { = Fluid; } \\
& \mathrm{i} \quad \text { = Inlet; } \\
& \text { int }=\text { Interface; } \\
& \mathrm{L}=\text { Local; } \\
& \mathrm{Lc}=\text { Local contribution; } \\
& \mathrm{s}=\text { Solid; } \\
& \mathrm{sf}=\text { Surface; and } \\
& \mathrm{x} \quad=\text { Phase }
\end{aligned}
$$




\section{Introduction}

Mixed convection heat transfer (or combined forced and free convection heat transfer) occurs when the buoyance force considerably affects forced convection. Practically, in industry, it takes place (or intentionally appears) when the desired heat cannot be transferred due to reasons such as high heat generation (or heat flux) and/or low required pump/fan power (or small space). Examples for occurrence of mixed convection heat transfer are mixed convection in electronic equipment (particularly with variable heat generation), in fuel assemblies of nuclear reactor cores, in solar collectors, etc. On the other hand, porous media (particularly high conductive porous media such as metal foams) becomes popular in heat transfer enhancement field due to high volumetric convective heat transfer coefficient and effective thermal conductivity. The analysis of mixed convection in heat transfer has taken the attention of researchers in recent years. Adequate knowledge on both mixed convection and heat transfer in porous media is essential to determine heat transfer rate in porous media under mixed convection.

Volume average method (VAM) is widely used to handle heat and fluid flow problems in porous media. The solution of volume averaged governing equations yields volume averaged velocity, temperature and pressure, which are imaginary quantities. In VAM, there are volume averaged transport properties (macroscopic transport properties) such as permeability, interfacial convective heat transfer coefficient, thermal dispersion and thermal tortuosity whose values must be known. The values of these macroscopic transport properties depend on the structure, thermophysical properties of porous media and flow field.

For the problem of local thermal non-equilibrium heat transfer in porous media, interfacial convective heat transfer coefficient must be known to calculate heat flux between fluid and solid phases. The interfacial convective heat transfer coefficient can be obtained numerically and/or experimentally. Recent developments in computational methods and computer technologies have enabled the determination of interfacial convective heat transfer coefficient, numerically. For instance, Kuwahara et al. (2001) numerically studied the interfacial convective heat transfer coefficient for forced convection in a periodic porous medium consisting of square rods with staggered arrangement under local thermal non-equilibrium. Similar studies for the effect of different parameters were performed by Nakayama et al. (2002), Ozgumus and Mobedi (2015), Petrasch et al. (2008), Saito and de Lemos (2005), Teruel and Díaz (2013) and Gamrat et al. (2008). Furthermore, Rees (2010) performed microscopic study on a composite solid saturated with a single fluid to calculate values for the interface heat transfer coefficient; however, the effect of fluid flow was not considered.

Our literature survey showed that many studies on mixed convection heat transfer in porous media under the assumption of local thermal equilibrium have been performed; hence, the interfacial convective heat transfer coefficient was not needed (Yang et al., 2016; Sheremet and Pop, 2015; Rosca et al., 2014; Oztop et al., 2012). However, there are limited studies in which the local thermal non-equilibrium approach is used and consequently the interfacial convective heat transfer coefficient must be known. Table I shows the list of some reported macroscopic studies under local thermal non-equilibrium assumption on mixed convection in porous media. In the first column of the table, the name and reference number of researcher are written. In the second and third columns, the governing equations used and definition of the dimensionless heat transfer coefficient are presented. Finally, in the fourth column, the range of dimensionless heat transfer coefficient is given.

Saeid (2004) analyzed mixed convection in a vertical porous layer under local thermal non-equilibrium condition. They defined a dimensionless interfacial heat transfer coefficient in terms of porosity and fluid thermal conductivity. The value of dimensionless interfacial heat transfer coefficient changes between 0.01 and 100. Wong and Saeid (2009) considered 



jet impingement cooling in a horizontal layer for mixed convection case under local thermal non-equilibrium condition. They used the same dimensionless interfacial heat transfer coefficient of Wong and Saeid (2009); however, the value of this parameter changed from 0.01 to 1,000 . Buonomo et al. (2010) studied on transient mixed convection in channels that were partially heated and filled with a porous medium in which a local non-thermal equilibrium approach was used. A correlation valid for forced convection interfacial heat transfer was used in their study. Ahmed et al. (2011) focused on mixed convection in an annular vertical cylinder filled with saturated porous medium with local thermal nonequilibrium model. The same definition of interfacial mixed convection heat transfer by Saeid (2004) and Wong and Saeid (2009) was used; however, the study was performed for three dimensionless interfacial mixed convection as 0.1, 1 and 100. Khandelwal and Bera (2012) studied on mixed convection in a vertical channel under the local thermal nonequilibrium condition. They used the same definition and range of dimensionless mixed convection heat transfer that was used in Saeid (2004). Buonomo et al. (2012) analyzed an open cavity that filled with porous media for transient mixed convection case. They preferred to use Biot number. Buonomo et al. (2014) also performed another study on mixed convection in channels partially heated at uniform heat flux filled with a porous medium under local thermal non-equilibrium condition. The same correlation used by Buonomo et al. (2010) was used in their study.

No doubt, the results of the above studies used the assumption of local thermal nonequilibrium model are valuable. However, in those studies, the dimensionless interfacial convective heat transfer coefficient varies between a possible minimum and maximum values, and no information was given about the practical value of the interfacial convective heat transfer coefficient. The above review on local thermal non-equilibrium model of mixed convection heat transfer in porous media shows that there is no clear idea about the value of the interfacial mixed convective heat transfer coefficient.

In this study, the interfacial mixed convective heat transfer coefficient for a periodic porous medium is determined numerically. The pore scale governing equations are written in dimensionless form and solved computationally for a representative volume. Based on the obtained results, the value of the interfacial convective heat transfer coefficient is calculated by using VAM. A detailed discussion on the effect of governing dimensionless parameters (porosity, Reynolds and Richardson numbers) on the local and average interfacial Nusselt number is done. To the best of our knowledge, there is no study on determination of interfacial convective heat transfer coefficient for mixed convection in porous media in literature. The present study might be the first study providing an accurate idea on the range of this important parameter, which will be useful particularly for researchers who study on mixed convection heat transfer in porous media, macroscopically.

\section{Pore scale equations and volume-averaged governing equations for mixed convection}

Pore scale governing equations are the conservation of mass, momentum and energy equations for fluid phase and conduction heat transfer equation for solid phase. For the considered problem by including Boussinesq's approximation, they can be written as follows:

$$
\nabla \cdot \vec{u}=0
$$

Heat transfer coefficient

2781 


$$
\begin{gathered}
\rho_{f}(\vec{u} \cdot \nabla) \vec{u}=-\nabla p+\mu \nabla^{2} \vec{u}+\vec{g} \beta\left(T^{f}-T_{r e f}^{f}\right) \\
\left.\left(\rho C_{p}\right)_{f} \overrightarrow{(u} \cdot \nabla\right) T^{f}=k_{f} \nabla^{2} T^{f} \\
\nabla^{2} T^{s}=0
\end{gathered}
$$

where $T^{s}$ and $T^{f}$ represent solid and fluid temperature at interface, respectively. Following Nakayama (1995), the above equations can be integrated over a control volume to obtain volume-averaged energy equations for fluid and solid phases. For a quantity in a representative elementary volume (REV) shown by $V$, two types of volume averaging exist:

$$
\begin{aligned}
\langle\phi\rangle & =\frac{1}{V} \int_{V} \phi d V \\
\langle\phi\rangle^{x} & =\frac{1}{V_{x}} \int_{V_{x}} \phi d V
\end{aligned}
$$

which are called as volume average and intrinsic volume average of $\phi$ quantity, respectively. By using these definitions and doing some mathematical manipulation, the steady-state volume averaged energy equation for solid and fluid can be yielded as follow:

$$
\begin{gathered}
\left(\rho C_{p}\right)_{f}(\langle\vec{u}\rangle \cdot \nabla)\langle T\rangle^{f}=\nabla \cdot\left[k_{f} \varepsilon \nabla\langle T\rangle^{f}+\frac{1}{V} \int_{A_{\text {int }}} k_{f} T^{f} d A-\left(\rho C_{p}\right)_{f}\left\langle T^{\prime} u^{\prime}\right\rangle^{f}\right] \\
-\frac{1}{V} \int_{A_{\text {int }}} k_{f} \nabla T^{f} \cdot \vec{n} d A \\
\nabla \cdot\left[k_{s}(1-\varepsilon) \nabla\langle T\rangle^{s}-\frac{1}{V} \int_{A_{\text {int }}} k_{s} T^{s} d A\right]-\frac{1}{V} \int_{A_{\text {int }}} k_{s} \nabla T^{s} \cdot \vec{n} d A=0
\end{gathered}
$$

where $A_{\text {int }}$ is the interface area between the fluid and solid, $\vec{n}$ is the normal vector pointing outward from the fluid to solid, $\langle T\rangle^{f}$ and $\langle T\rangle^{s}$ are intrinsic volume average of the fluid and solid side, respectively. For equation (7), the left side term is heat transfer by convection, the first term on the right side is heat transfer by conduction, the second term shows thermal tortuosity, the third term refers to thermal dispersion and the fourth term is interfacial convective heat transfer. For equation (8), the first term is diffusion, while the second one is thermal tortuosity; finally, the third term refers to heat transfer between solid and fluid phase.

Discussions on the above terms have been performed in details in study of Ozgumus et al. (2013). As it was mentioned above, the last term in equations (7) and (8) refers heat transfer between solid and fluid phases in porous media. To determine its value, the interfacial convective heat transfer coefficient between solid and fluid is defined:

$$
h_{i n t}=\frac{\frac{1}{V} \int_{A_{\text {int }}} k_{f} \nabla T_{f} \cdot \vec{n} d A}{A_{s s}\left(\langle T\rangle^{s}-\langle T\rangle^{f}\right)}
$$


where $h_{\text {int }}$ is the interfacial convective heat transfer coefficient and $A_{s s}$ is the solid-fluid interface area. Hence, to predict the volume average temperature for solid and fluid phases, the value of $h_{\text {int }}$ should be known.

As it was mentioned before, the dimensionless form of the pore scale governing equations is solved to obtain velocity and temperature field in the voids. The dimensionless pore scale governing equations are:

$$
\begin{gathered}
\nabla^{*} \cdot \vec{u}^{*}=0 \\
\vec{u}^{*} \cdot \nabla^{*} \overrightarrow{u^{*}}=-\nabla^{*} p^{*}+\frac{1}{\operatorname{Re}} \nabla^{* 2} \overrightarrow{u^{*}}+\operatorname{Ri} \theta \vec{j} \\
\vec{u}^{*} \cdot \nabla^{*} \theta_{f}^{*}=\frac{1}{\operatorname{Re} \operatorname{Pr}} \nabla^{* 2} \theta_{f}^{*} \\
\nabla^{* 2} \theta_{s}^{*}=0
\end{gathered}
$$

where the dimensionless parameters used to find the above equations are defined as:

$$
\begin{aligned}
R e & =\frac{\left\langle\mathrm{v}_{i}\right\rangle w}{\nu \varepsilon} ; \quad G r=\frac{g \beta \Delta T w^{3}}{\nu^{2}} ; \nabla^{*}=\nabla / H, \vec{u}^{*}=\vec{u} /\left\langle\mathrm{v}_{i}\right\rangle, \quad P=\frac{p H}{v_{i} \mu_{f}} \\
\theta & =\frac{T-T_{i}}{T_{s}-T_{i}} ; \quad R i=\frac{G r}{R e^{2}}
\end{aligned}
$$

$P$ and $\theta$ are the dimensionless pressure and temperature of the fluid, $\left\langle\mathrm{v}_{i}\right\rangle$ is the inlet Darcy velocity. $H$ and $w$ are the width and length of a cell, and they are equal to each other in this study. $R e, R i$ and $\varepsilon$ are the main parameters which their effects are investigated in this study, while $\operatorname{Pr}$ is taken constant as 0.71 .

As can be seen from equation (9), the interfacial heat transfer coefficient is found based on temperature difference between $\langle T\rangle^{s}$ and $\langle T\rangle^{f}$. For a particle in porous media, the pore scale Biot number can be defined as:

$$
B i=\frac{h_{s f} l}{k_{s}}
$$

where $h_{s f}, l$ and $k_{s}$ are defined as solid-fluid surface interfacial heat transfer coefficient, characteristic length of particle and thermal conductivity of solid phase. The value of $l$ (which can be particle size) is small and $k_{s}$ is high for many solids in practical applications such as metals (in metal foams) and stones (in rock). Hence, $B i$ number takes small values (i.e. $B i \ll 1$ ) permitting researchers to apply constant temperature assumptions for solid phase. Hence, there might be no need to solve heat conduction equation for solid. The same assumption has been used by many researchers such as Nakayama et al. (2002), Ozgumus and Mobedi (2015) and Saito and de Lemos (2006). 
$\mathrm{HFF}$

27,12

2784

Figure 1.

The considered and studied domain

\section{The considered porous media and studied domain}

The schematic view of the considered porous media is shown in Figure 1. The studied domain consists of infinite square blocks in horizontal direction, while number of rods in vertical direction is 10 . The axial length of rods in z-direction is assumed large; therefore, two-dimensional flow assumption can be used. The porous medium has periodic structure in x-direction and a REV bounded by dash line, as shown in Figure 1, can be studied to obtain results for entire domain. The radiation heat transfer and viscous dissipation are neglected and gravity acts in $-\mathrm{y}$ direction. To prevent computational difficulties at the outlet boundary of porous media (due to possible reverse flows), an extension region is considered on the top of blocks. The ratio between $\mathrm{L}$ and $\mathrm{H}$ (i.e. $\mathrm{L} / \mathrm{H})$ is 19. The local and average interfacial heat transfer coefficient between the solid rods and its surrounded fluid is obtained for five porosity values $(0.51,0.64,0.75,0.84$ and 0.96$)$ and different values of Ri number (i.e. from 0.01 to 10 ) and Re number (i.e. from 100 to 500). As mentioned before, the study is performed for air with Prandtl number of 0.71 .

\section{Boundary conditions and calculation of interfacial Nusselt number}

Figure 2 shows the computational domain. By considering, $\vec{u}^{*}=u^{*} \vec{i}+\mathrm{v} \vec{j}$ where $u^{*}$ and $\mathrm{v}^{*}$ are dimensionless velocity components, the boundary conditions for the set of equations (10)-(12) mathematically can be expressed as follows:

For solid-fluid interface boundary:

$$
u^{*}=v^{*}=0 ; \quad \theta=1
$$

For left and right symmetry boundaries:

$$
\frac{\partial u^{*}}{\partial X}=\frac{\partial v^{*}}{\partial X}=\frac{\partial \theta}{\partial X}=0
$$

For inlet boundary:

$$
u^{*}=0 ; \quad v^{*}=1 ; \quad \theta=0
$$




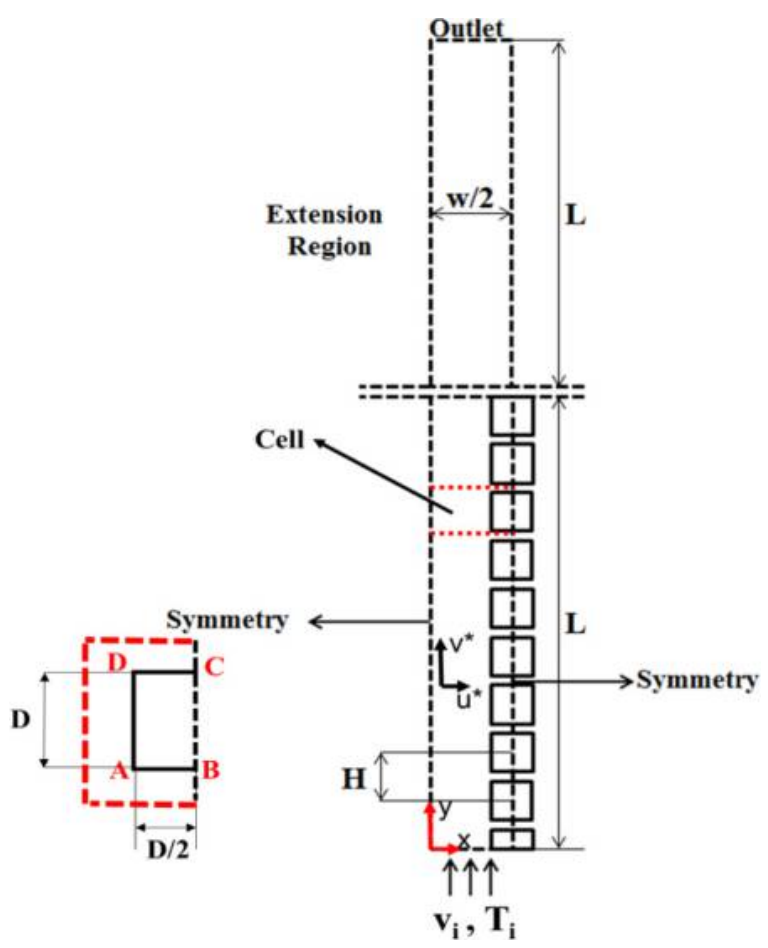

Heat transfer coefficient

2785

Figure 2.

A view of the computational domain with boundary conditions

For outlet boundary:

$$
\frac{\partial u^{*}}{\partial Y}=\frac{\partial v^{*}}{\partial Y}=\frac{\partial \theta}{\partial Y}=0
$$

After determination of dimensionless temperature, the velocity and pressure under the above boundary conditions, equation (9) is used to obtain intrinsic volume average of quantity $\langle T\rangle^{f}$ for each cell, schematically shown in Figure 2. Furthermore, it is possible to rewrite equation (9) in the following form by using dimensionless temperature defined in equation (14):

$$
\frac{h_{\text {int } H} H}{k} \frac{\left(\langle T\rangle^{f}-\left\langle T_{i}\right\rangle^{f}\right)}{\left(\langle T\rangle^{s}-\left\langle T_{i}\right\rangle^{f}\right)}=\frac{1}{A_{s f}^{*}} \int_{A_{s f}^{*}} \frac{\partial \theta}{\partial n^{*}} d A^{*}
$$

where $A^{*}$ and $n^{*}$ are dimensionless area and dimensionless normal direction. After some mathematical manipulations, the dimensionless local interfacial Nusselt number for a rod of the porous media can be obtained as: 


$$
N u_{L}=\frac{\frac{1}{A_{s f}^{*}} \int_{A_{s f}^{*}} \frac{\partial \theta}{\partial n^{*}} d A^{*}}{1-\langle\theta\rangle^{f}}
$$

Furthermore, the local Nusselt number for three different surfaces of rods $\left(\overline{N u}_{L c}\right)$ in REV is also calculated to find out the effect of each surface on the heat transfer coefficient. $\overline{N u}_{L c}$ is called as face local interfacial Nusselt number in this study. For instance, for surface which is shown by $\mathrm{AB}$ in Figure 2, mathematically, it can be defined as:

$$
\left.\bar{N} u_{L c}\right|_{A B}=\frac{\frac{1}{|A B|} \int_{A}^{B} \frac{\partial \theta}{\partial n^{*}} d A^{*}}{1-\langle\theta\rangle^{f}}
$$

where $\left.\bar{N} u_{L C}\right|_{A B}$ is the average of Nusselt number on the surface of AB. If the same equation is applied to the surface of $\mathrm{BC}$ and $\mathrm{CD}$ of Figure 2, the summation of the obtained values will be equal to the result of equation (14), as the denominator is defined based on the volume averaged-temperature between fluid and solid in the cell, and it is identical with the summation of face local interfacial Nu number of three surfaces:

$$
\bar{N} u_{L}=\left.\bar{N} u_{L c}\right|_{A B}+\left.\bar{N} u_{L c}\right|_{B C}+\left.\bar{N} u_{L c}\right|_{C D}
$$

The above equation is used only to show the effect of each surface of the rods on the local Nusselt number and does not have any practical usage.

The average interfacial Nusselt number of the porous media is calculated by Nusselt number. The average interfacial $\mathrm{Nu}$ number of porous media is calculated by taking the arithmetical average of the local Nusselt number values of each rod from 2 to 10 in $\mathrm{y}$ direction and it can be expressed as:

$$
\overline{N u}=\frac{\sum_{i=2}^{i=10} N u_{L}(i)}{N}
$$

where $N$ is number of blocks in fully developed region. $N$ is considered as 9 in this study, which is number of rods from 2 nd to 10 th.

\section{The numerical procedure and computational details}

Pore scale governing equations are solved for the considered REV shown in Figure 2, by a commercial finite volume code, ANSYS Fluent 15.0. The power law scheme is used for the discretization of the convection terms in the momentum and energy equations. Semi-implicit method for pressure linked equations (SIMPLE) is used to handle the pressure-velocity coupling. The absolute criteria based the convergence criterion is set to $10^{-7}$ for continuity and the momentum equations, $10^{-12}$ for the energy equation. Furthermore, the velocity and 
temperature profiles at $\mathrm{Y}=3,6$ and 9 are checked for different iteration steps and the solution is accepted as converged when no change for these profiles is observed. As it was mentioned before, an extension region is considered above the porous region to prevent computational difficulties at the outlet of porous region due to reverse flows. Our computational experience showed that the length of $\mathrm{L}$ is sufficient for the height of dummy region. Grid independency study is done for two limit cases of this study as $\mathrm{Ri}=0.01, \mathrm{Re}=$ 100 and $\mathrm{Ri}=10, \mathrm{Re}=500$ and for porosity of 0.96 where this configuration has the most complex flow for the considered problem. The grid number in $\mathrm{X}$ direction is taken from 10 to coefficient 260, while in Y direction, it changes from 190 to 4,560, respectively. The interfacial $\mathrm{Nu}$ number is calculated and compared for the considered different number of grids. The results are presented in Figure 3(a). The average interfacial Nu number does not highly change after grid number of $220 \times 4,180$. It is observed that the grid number of $260 \times 4,940$ is sufficient to obtain accurate results and observe flow reversals in the channel in details.

\section{Results and discussion}

In this section, after validating of the numerical study, the change of velocity and temperature fields with governing dimensionless parameters are described. Then, the

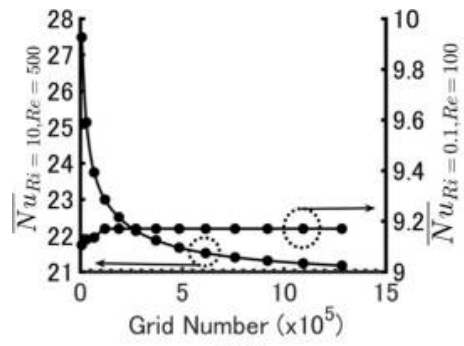

(a)

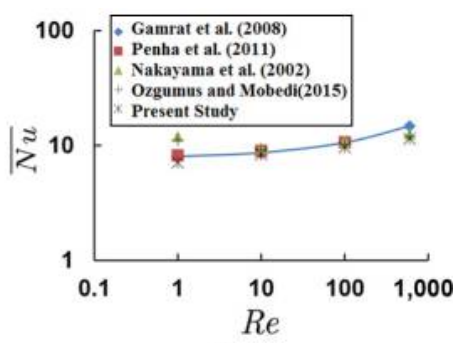

(b)

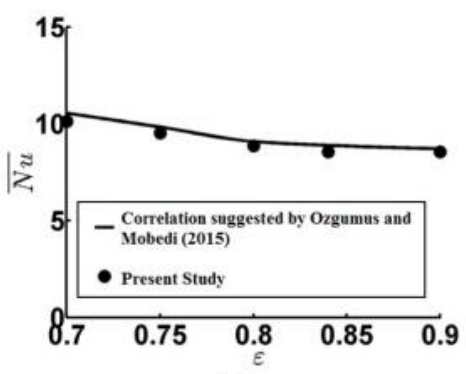

(c)

Notes: (a) grid independence study for $\mathrm{Ri}=0.0001$ and $\varepsilon=0.75$ when $\varepsilon=0.96$; (b) the comparison of present study with reported results for average interfacial Nusselt number of fully developed forced convection; (c) comparison of average interfacial Nusselt number with correlation suggested by Ozgumus and Mobedi (2015) when $\mathrm{Ri}=0.0001$ and $\mathrm{Re}=$ 100

Figure 3. Validation of the obtained results 
HFF

27,12

2788

change of local and average Nusselt number with the same dimensionless parameters is analyzed.

\subsection{Validation of obtained numerical results}

The code and method used in this study are validated by using the reported studies in literature. As it was mentioned before, most of studies on interfacial convective heat transfer coefficient were performed for forced convection in porous media. Figure 3(b) shows the comparison of the present study with the reported results of the fully developed forced for a porous media consisting of square rods and has porosity of 0.75 . The comparison is accomplished for Ri number of 0.0001 presenting a forced convection heat transfer. The present results are compared with the results reported by Ozgumus and Mobedi (2015), Penha et al. (2011), Gamrat et al. (2008) and Nakayama et al. (2002). A good agreement between the present results and those reported studies can be observed. The results of the present study are also validated by comparing with the relationship suggested in the study of Ozgumus and Mobedi (2015) as shown in Figure 3(c). The correlation in their study was given for forced convection heat transfer when Re changes between 1 and 100. The correlation is valid for porosity values between 0.7 and 0.90 . A good agreement between the suggested correlation and the present study for $\mathrm{Ri}=0.0001$ and $\mathrm{Re}=100$ can be observed.

\subsection{Velocity and temperature fields}

Figure 4 shows the velocity and the temperature fields for a mixed convection heat transfer in the porous media with $\varepsilon=0.96, \mathrm{Ri}=0.01, \mathrm{Re}=100$. Figure 4 (a) shows the streamline and temperature distribution for heat and fluid flow with $\mathrm{Ri}=0.01$ and $\mathrm{Re}=100$. For better visualization of streamline and temperature contours, the width of the channel is extended by two times. Due to the low Ri number and buoyancy effect, the forced convection is dominant, and no reverse flow occurs inside the porous media. The fluid flows straightly upward in the center of the voids between the blocks, while the air which is close to the blocks enters into the gap between them. A close-up view of the flow around seventh rod can be also seen in the same figure. A secondary flow occurs on the top of each rod causing the reduction of heat transfer rate from the top surface of rod to the surroundings. The value and contribution of face local interfacial Nusselt number of each faces (i.e. A - B, B - C and C - D) of seventh rod onto $\bar{N} u_{L}$ can be seen in the table of Figure 4(a). As it can be seen from the table, heat is mainly transferred from the surface B-C and the effect of the surface C-D to the local interfacial Nusselt number is small compared to the other surfaces due to the occurrence of secondary loop.

By increasing of Re number from 100 to 500, no significant change in the general behavior of the flow is observed due to low Ri number as can be seen from Figure 4(b). For $\operatorname{Re}=500$, the flow between the blocks becomes straighter and vortices occurring in the vertical gaps between blocks become larger. The comparison between the temperature contours of porous media of $\operatorname{Re}=100$ and 500 [Figure 4(a) and (b)] shows that the cold fluid moves long distance in vertical direction. It should be mentioned that heat transfer coefficient is defined based on the surface and inlet temperature difference. That is why the interfacial convective heat transfer coefficient may be expected to be higher for a flow with $\operatorname{Re}=500$ compared to $\operatorname{Re}=100$. The contribution of $\bar{N} u_{L c}$ of each face of seventh rod to the local interfacial Nusselt number is given in table of Figure 4(b). By increasing Re number from 100 to 500, there is nearly no considerable change in the contribution of $\bar{N} u_{L c}$ from surfaces, just the contribution from surface B-C a little bit increases, while the contribution of surface C-D decreases compared to the case of Figure 4(a). 


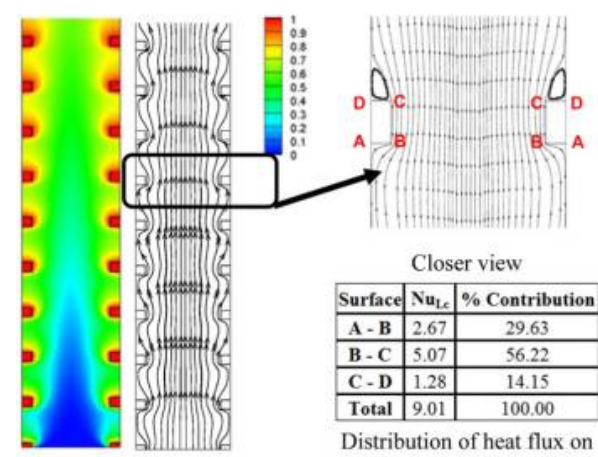

(a)

the surface

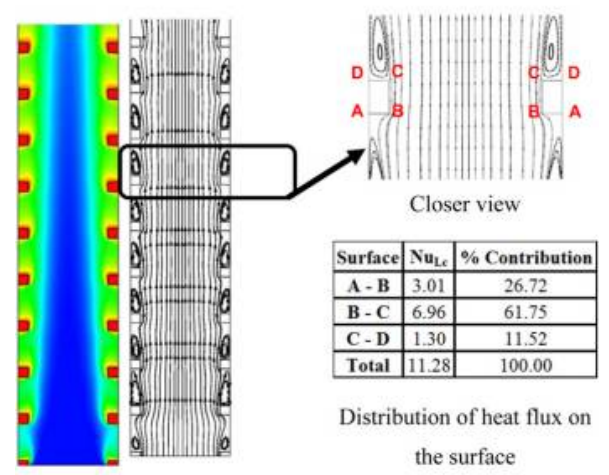

(b)

Notes: (a) $\operatorname{Re}=100$; (b) $\operatorname{Re}=500$
Heat transfer coefficient

2789
Figure 4.

Streamlines and temperature distribution in porous media with $\varepsilon=0.96$ and $\mathrm{Ri}=0.01$

The structure of porous media in Figure 5(a) is the same with Figure 4(a); however, the Ri number is increased from 0.01 to 10 . Similarly, the width of the channel in the figure is extended two times for better visualization. Figure 5(a) shows the flow and temperature distributions for $\operatorname{Re}=100$. As it can be seen, by increasing of Ri number from 0.01 to 10 , reverse flows in the middle of the channel occur due to the strong buoyancy effect. A closeup picture of the flow reversal for the fourth rod of the channel is given in Figure 5(a). The shape of the secondary flows in the middle and top of the rods can be observed clearly. The contribution of each surfaces of seventh rod on heat transfer rate from rod to air when $\mathrm{Ri}=$ 10 and $\operatorname{Re}=100$ shown in Table of Figure 5(a). Again, the same contribution observed for previous figures can be seen also for Figure 5(a).

The flow and temperature fields for $\mathrm{Re}=500$ and $\mathrm{Ri}=10$ are shown in Figure 5(b). By increasing of Re from 100 to 500, the size of reverse flows becomes larger both in longitudinal and transverse directions but occurs at a little bit far from the inlet region. The comparison between Figure 5(a) and (b) indicates that the cold fluid moves longer distance in vertical direction, and the interfacial convective heat transfer coefficient is expected to be increased when the Re value is increased from 100 to 500. The interesting point of Figure 5 (b) is a secondary flow occurs on the vertical wall of the rods (face BC). Hence, this secondary 
HFF

27,12

2790

\section{Figure 5.}

Streamlines and temperature distribution in porous media with $\varepsilon=0.96$ and $\mathrm{Ri}=10$

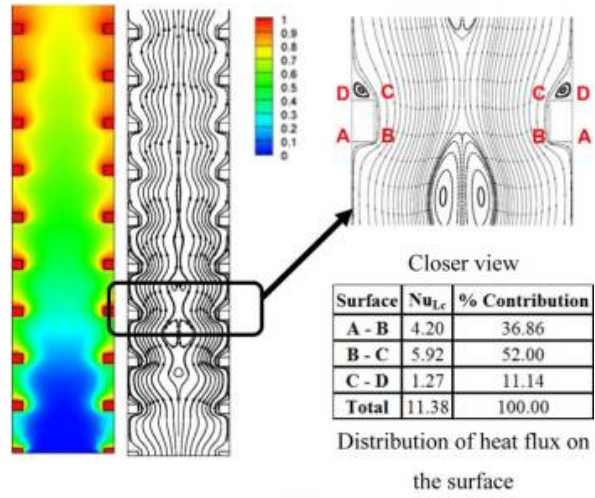

(a)

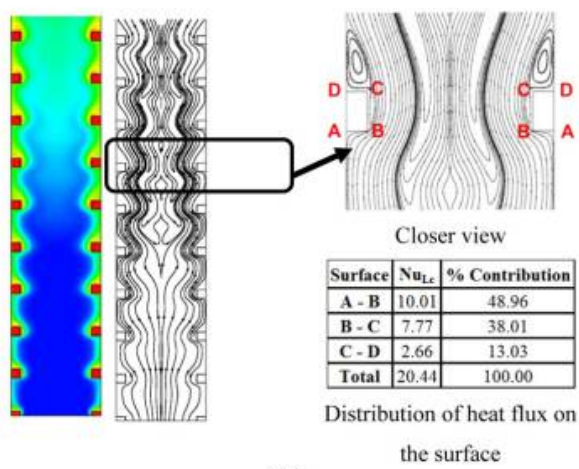

(b)

Notes: (a) $\operatorname{Re}=100$; (b) $\operatorname{Re}=500$

flow may reduce heat transfer coefficient on the $\mathrm{BC}$ surface. Similar to the previous figures, the contribution of each surface on the interfacial heat transfer coefficient is shown in the same figure. It is seen that the heat transfer from surface B-C decreases as compared to Figure 5(a), as expected. As secondary loop occurs near the surface B-C, and heat is mainly transferred from surface A-B, and surface C-D still has the lowest effect on the interfacial heat transfer coefficient.

Figure 6 shows the streamline and temperature contours for the porous media with porosity of 0.51 when $\mathrm{Ri}=0.01$. Figure 6 (a) shows the temperature field and streamline for $\operatorname{Re}=100$. Similar to the Figure 4(a), due to the low buoyancy effect, the streamlines are straight, and no flow reversal occurs in the flow direction, as forced convection is dominant heat transfer mechanism. Secondary flow occurs not only on the top but also on the bottom side with equal area. Due to high heat transfer area between the solid and fluid phases, the temperature of fluid that enters the porous media attains to the solid temperature faster than Figures 4(a) in which $\varepsilon=0.96$. Another interesting point of Figure 6(a) is the contribution of heat transfer of each surface into the total heat transfer rate from rod. The surface B-C is the most important surface with 80.76 per cent, while 


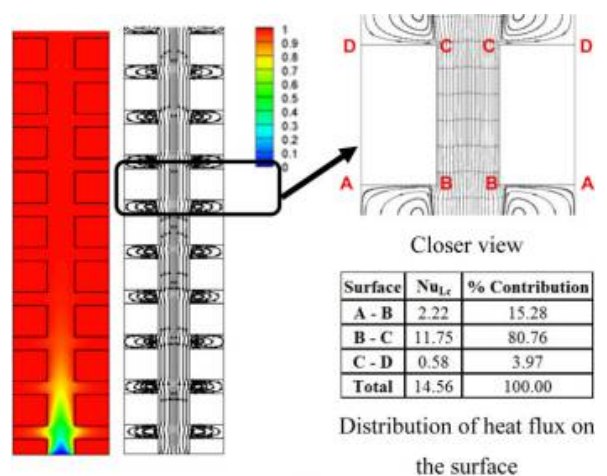

(a)

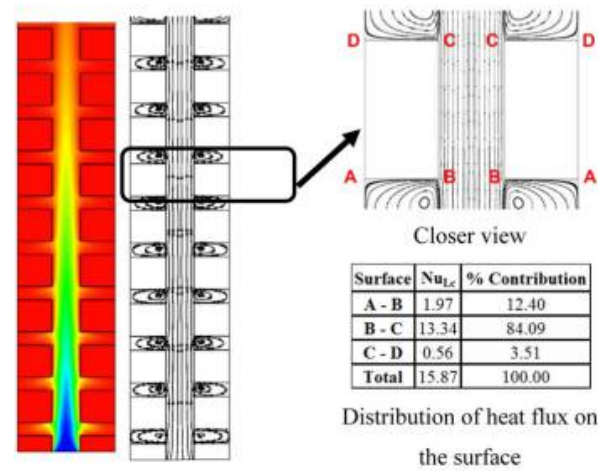

(b)

Notes: (a) $\operatorname{Re}=100$; (b) $\operatorname{Re}=500$
Heat transfer coefficient

2791

Figure 6.

Streamlines and temperature distribution in porous media with $\varepsilon=0.51$ and $\mathrm{Ri}=0.01$

the effect from surfaces A-B and C-D totally is 19.26 per cent. By increasing of Re number from 100 to 500 , there is no significant change in the velocity field inside the channel as seen from Figure 6(b). However, the cold fluid entering to the porous media moves longer distance in vertical direction due to high value of $\operatorname{Re}$ number (i.e. $\operatorname{Re}=$ 500 ), and then higher interfacial Nusselt value may be expected for $\operatorname{Re}=500$. There is no considerable change in contribution from each surface when it is compared with Figure 6(a), as it can be seen from the table in Figure 6(b).

Figure $7(a)$ and (b) shows the streamline and temperature field for the same porosity of Figure 6(a) and (b), when $\mathrm{Ri}=10$ and $\mathrm{Re}=100$ and 500, respectively. No significant changes are observed in the flow and temperature field if Ri number is increased by 1,000 times. The only change of flow configuration is the number of secondary flows for $\operatorname{Re}=500$ [Figure 7(b)] occurring in the space between the rods in vertical direction. However, the top and bottom surface does not have an important effect on the total heat transfer rate from the rod. Figures 6 and 7 show that for a porous medium with low porosity, the streamline and temperature contours in most regions of porous medium are not changed considerably by the change in Re or Ri numbers. 
HFF

27,12

2792

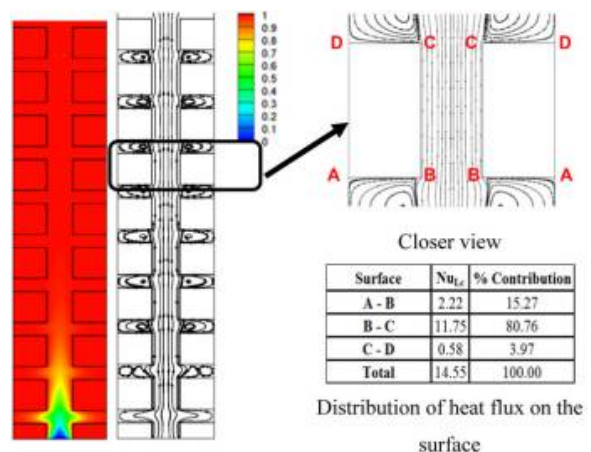

(a)

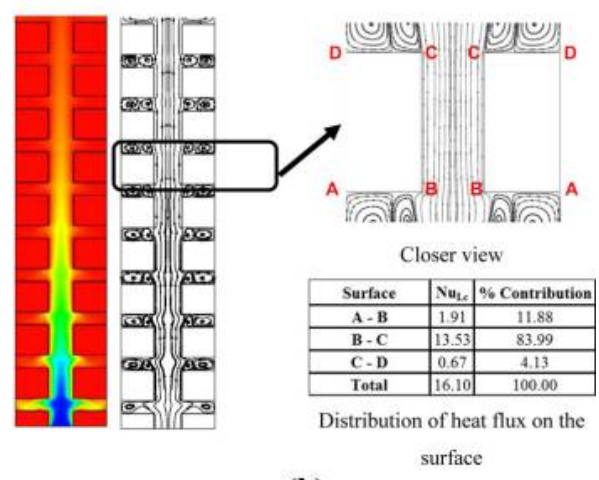

(b)

Notes: (a) $\operatorname{Re}=100$; (b) $\operatorname{Re}=500$

\subsection{Local interfacial convective heat transfer coefficient}

Figure 8 shows the change of local interfacial Nusselt number with Reynolds number for different values of porosity and Ri. The change of local interfacial Nusselt number with Re number for $\mathrm{Ri}=0.01$ and $\varepsilon=0.96$ is shown in Figure 8(a). For low value of Ri number (i.e. $\mathrm{Ri}=0.01$ ), the buoyancy effect is negligible, and forced convection heat transfer is dominant. As it can be seen from Figure 8(a), the value of $N u_{L}$ is high for the first block and then it decreases steeply and takes almost a constant value for the rest of blocks showing fully developed condition. For $\operatorname{Re}=100$, the flow attains to the fully develop condition faster than $\operatorname{Re}=500$, as it is expected. In general, Figure 8(a) shows that when Ri number is small (e.g. $\mathrm{Ri}=0.01$ ), the interfacial convective heat transfer coefficient does not change considerably with Reynolds number, particularly for the blocks in fully developed regions. A slight of increase of $N u_{L}$ with increase of Re is observed for fully developed condition. For instance, the rate of increase of $N u_{L}$ of tenth rod of porous media with $\mathrm{Ri}=0.01$ and $\varepsilon=0.96$ when Re number increases from 100 to 500 is around 20 per cent. By increasing Ri number from 0.01 to 10, the local interfacial Nusselt number increases with Re number, as can be seen from Figure 8(b). The reverse flows occur in the middle of the channel, and this causes the mixing of flow and increase of local interfacial Nusselt number. By the increase in Re number, the size of the secondary flows at the middle of the channel increases [Figures 5(a) 


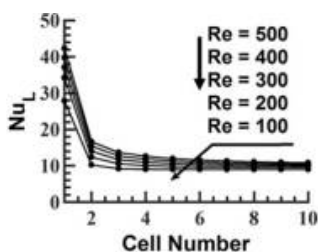

(a)

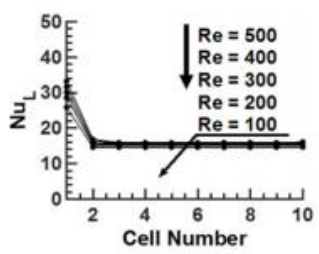

(c)

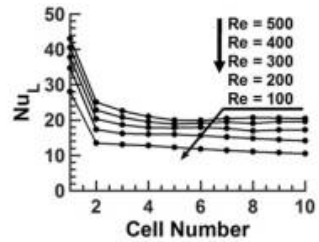

(b)

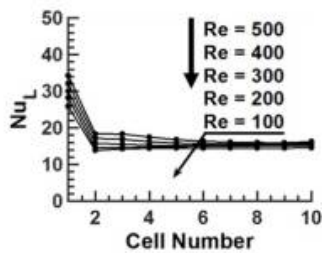

(d)

Notes: (a) $\mathrm{Ri}=0.01, \varepsilon=0.96$; (b) $\mathrm{Ri}=10, \varepsilon=0.96$; (c) $\mathrm{Ri}=0.01, \varepsilon=0.51$; (d) $\mathrm{Ri}=10, \varepsilon=0.51$

Heat transfer coefficient

2793

Figure 8.

The change of local interfacial Nusselt number distribution with porosity, Re and Rinumbers

and (b)], and that is why an obvious difference between the local Nusselt numbers of channels with different Reynolds number is seen which is completely different than the one in Figure 8(a). The increase in size and mixing effect of the secondary flows also increases the interfacial heat transfer coefficient.

By decreasing porosity from 0.96 to 0.51 , as shown in Figure 8(c), there is almost no change in the general behavior of interfacial Nusselt number with Reynolds number. When $\mathrm{Ri}=0.01$, the interfacial convective heat transfer coefficient of the rods is very close to each other for different Reynolds number, as the increase in Reynolds number in the porous media does not change the behavior of flow (Figures 6-7). For the porous media with $\varepsilon=$ 0.51 and $\mathrm{Ri}=10$, as it is shown in Figure 8(d), the local interfacial Nusselt number increases with Re number for the first two or third blocks; then again, the values of interfacial convective heat transfer coefficient come close to each other. Figure 8 clearly shows the effect of porosity on the heat and fluid flow in the mixed convection. The rate of increase of $N u_{L}$ for the tenth rod when Re number increases from 100 to 500 for a porous media with $\mathrm{Ri}=10$ and $\varepsilon=0.96$ is 95 per cent [Figure 8(b)], while for a porous media with $\varepsilon=0.51$ under the same condition, the rate of increase is 11 per cent [Figure 8(d)].

Figure 9 compares the distribution of local Nusselt number of the mixed convection with forced convection for two Ri number of 0.01 and 10, two Re numbers of 100 and 500 and, finally, two porosities of 0.51 and 0.96 . As can be seen from this figure, the forced convection interfacial heat transfer coefficient is very close to the mixed convection, except for the porous media with high porosity (i.e. $\varepsilon=0.96$ ) and Ri number (i.e. Ri $=10$ ). For high porosity values, the value of $N u_{L}$ deviates from forced convection, and the rate of increase for $N u_{L}$ increases with an increase in Ri number. For instance, the values of $N u_{L}$ for the last rods of channels increase by 100 per cent, if the Ri number increases from 0 (forced convection) to 10.

\subsection{Mean interfacial convective heat transfer coefficient}

Figure 10 shows the change of mean interfacial Nusselt number with Re and Ri numbers for different porosity values. The value of $\bar{N} u_{L}$ for forced convection is also plotted in the same 
HFF

27,12

2794

Figure 9.

The local interfacial Nusselt number between forced and mixed convection for two values of $\varepsilon$, Re and Ri numbers

Figure 10.

The change of mean interfacial Nusselt number distribution with porosity and Ri number (a) $\operatorname{Re}=100$; (b) $\operatorname{Re}=500$.

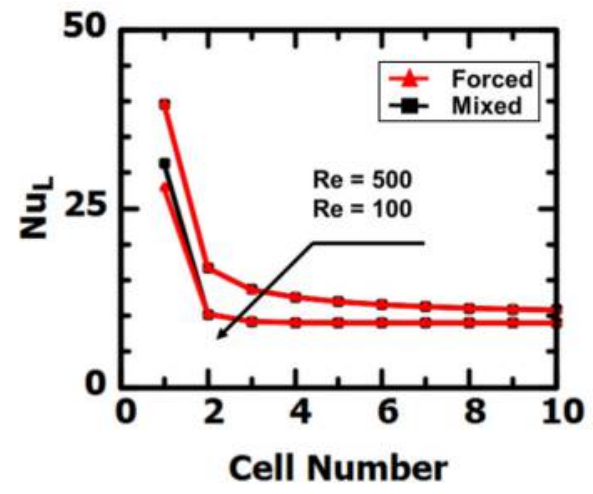

(a)

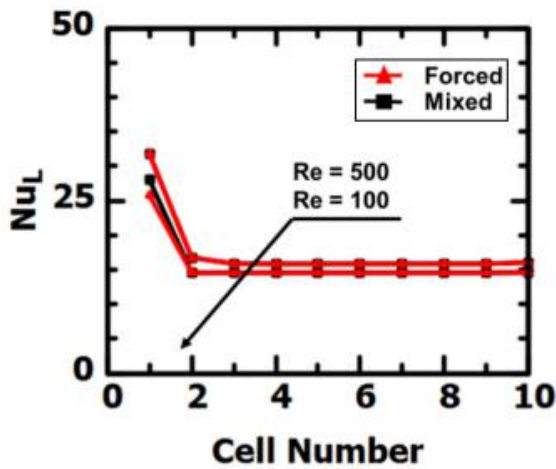

(c)

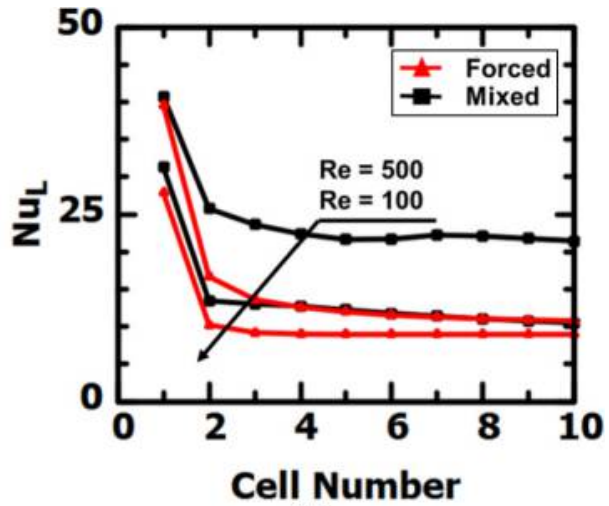

(b)

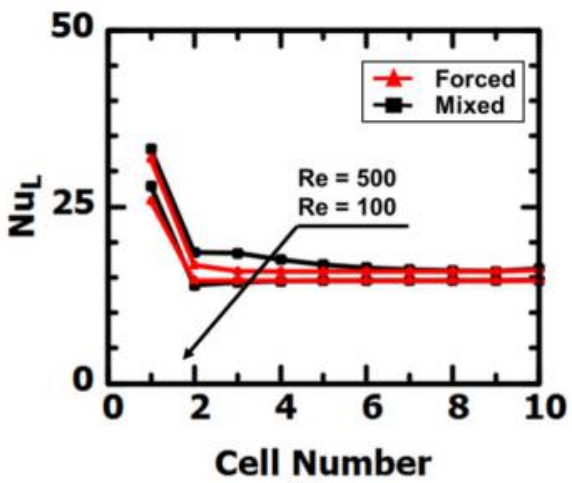

(d)

Notes: (a) $\mathrm{Ri}=0.01, \varepsilon=0.96$; (b) $\mathrm{Ri}=10, \varepsilon=0.96$; (c) $\mathrm{Ri}=0.01, \varepsilon=0.51$; (d) $\mathrm{Ri}=10, \varepsilon=$ 0.51

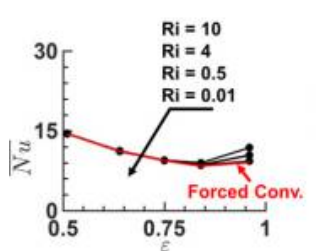

(a)

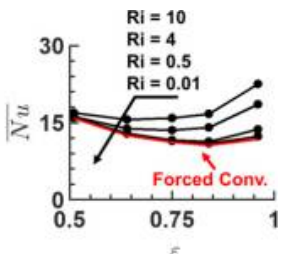

(b)

Notes: (a) $\operatorname{Re}=100$; (b) $\operatorname{Re}=500$

figure. The change of mean interfacial Nusselt number with porosity for different Ri numbers when $\mathrm{Re}=100$ is shown in Figure 10(a). As it can be seen, the interfacial Nusselt number has almost the same value for $\varepsilon=0.51$ (as expected), and it decreases with the increase in porosity. After a specific porosity, the interfacial convective heat transfer 
coefficient increases. The rate of increase depends on the Ri number. For small values of Ri number (e.g. $\mathrm{Ri}=0.01$ ), the rate of increase in interfacial convective heat transfer coefficient is small, while for high values of Ri number (e.g. Ri $=10$ ), the rate of increase becomes considerable due to the increase of buoyancy effect. For example, by an increase in the Ri number from 0.01 to 10 for $\operatorname{Re}=100$, the change of $\bar{N} u$ for a porous medium with $\varepsilon=0.51$ is 0.61 per cent, while for a porous medium with $\varepsilon=0.96$ under the same condition, the rate of increase is 29 per cent. Similar comments can be made for $\mathrm{Re}=500$ case, as shown in Figure 10(b). However, the rate of increase of interfacial convective heat transfer coefficient with Ri number becomes stronger. Particularly, for high porosity values (such as $\varepsilon=0.96$ ), the increase in the size of secondary flows in the middle of channel due to the increase of buoyancy effect causes the considerable increase in heat transfer between solid and fluid. For instance, for a porous media with $\varepsilon=0.96$, the rate of increase of $\bar{N} u$ for $\operatorname{Re}=500$ when $\mathrm{Ri}$ changes from 0.01 to 10 is 72 per cent. The trend for enhancement of heat transfer by including buoyancy effect can also be observed from Figure 10. The value of interfacial heat transfer coefficient of forced convection flow can considerably increase for porous media when porosity is high $(\varepsilon=0.96)$ and $\mathrm{Ri}$ increases from 0 to 10 .

Figure 11 shows the change of $\bar{N} u$ with Ri and Re numbers for two values porosity as 0.51 and 0.96 . The change of $\bar{N} u$ with Re numbers when $\varepsilon=0.51$ is shown in Figure 11(a). As it can be seen in Figure 11(a), $\bar{N} u$ does not change significantly by an increase in Ri number even for high values of Re number, as there is not sufficient void for reverse flow when porosity is low. That is why, the change in Re or Ri number has nearly no effect on heat and fluid flow when porosity is low. By increasing porosity value from 0.51 to 0.96 as shown in Figure 11(b), $\bar{N} u$ increases with Ri number; however, the rate of increase depends on the Re number. For $\operatorname{Re}=100$, the rate of increase is small, while it reaches to the highest increasing rate for $\mathrm{Re}=500$. As it was explained before, the effect of buoyancy on the interfacial heat transfer coefficient can be observed only for porous media with high values of porosity and Ri number.

\section{Conclusion}

In this study, the local and average interfacial convective heat transfer coefficient of a periodic porous medium under mixed convection heat transfer is determined computationally by using volume-averaging method. The pore scale governing equations are solved, and based on the obtained temperature distribution, the interfacial convective heat transfer coefficient is calculated. Based on the obtained results, following remarks can be concluded:

- The influence of the surface parallel to flow is higher for the porous media with low values of porosity (i.e. $\varepsilon=0.51$ ). For porous media with high porosity, Re and Ri

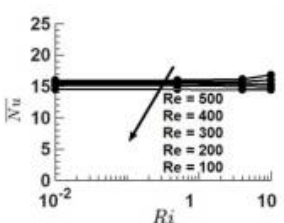

(a)

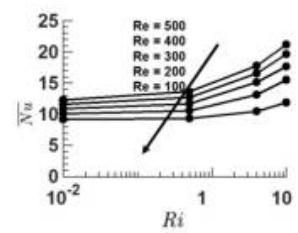

(b)

Notes: (a) $\varepsilon=0.51$; (b) $\varepsilon=0.96$

Heat transfer coefficient

2795
Figure 11.

The change of mean interfacial Nusselt number distribution with $\mathrm{Ri}$ and $\mathrm{Re}$ numbers (a) $\varepsilon=0.51$; (b) $\varepsilon=0.96$ 
HFF

27,12

numbers $(\varepsilon=0.96, \operatorname{Re}=500, \mathrm{Ri}=10)$, a secondary flow occurs on the surface parallel to flow, and bottom surface of the rod plays important role on the interfacial heat transfer coefficient.

- For low porosity values (i.e. $\varepsilon=0.51$ ), the mean interfacial Nusselt number does not change significantly with Ri or Re numbers. The rate of increase in mean interfacial convective heat transfer coefficient for a porous medium with $\varepsilon=0.51$ and $\operatorname{Re}=$ 100 , when Ri increases from 0.01 to 10 is only 0.64 per cent.

- For the porous media with high values of porosity (i.e. $\varepsilon=0.96$ ), the interfacial convective heat transfer coefficient considerably improves with increase of $\mathrm{Ri}$ number. However, the rate of increase depends on the Re number. For a porous medium with high porosity values, secondary flows occur in the main channel between rods when $\mathrm{Ri}$ value is high (i.e. $\mathrm{Ri}=10$ ), and this enhances heat transfer rate. For a porous medium with $\varepsilon=0.96$ and $R e=500$, the value of mean interfacial convective heat transfer coefficient improves by 72 per cent when Ri changes from 0.01 to 10 .

- The available correlations used for determination of interfacial heat transfer coefficient for forced convection can be used for mixed convection for low porosity (such as $\varepsilon=0.51$ ) even for high Re and Ri numbers. However, those correlations are not valid for porous media with high porosity values (such as $\varepsilon=0.96$ ) and Ri numbers $(\mathrm{Ri}=10)$.

There is no doubt that the height of the porous medium has an important effect on the interfacial heat transfer coefficient when the buoyancy effect is considerable. For further study, the effect of height of channel and Pr number on the trend of change of interfacial heat transfer coefficient should be investigated.

\section{References}

Ahmed, N.S., Badruddin, I.A., Kanesan, J., Zainal, Z. and Ahamed, K.N. (2011), "Study of mixed convection in an annular vertical cylinder filled with saturated porous medium, using thermal non-equilibrium model", International Journal of Heat and Mass Transfer, Vol. 54 No. 17, pp. 3822-3825.

Buonomo, B., Manca, O., Mesolella, P. and Nardini, S. (2010), "Transient mixed convection in channels partially heated filled with a porous medium in non-local thermal equilibrium", Porous Media and Its Applications in Science, Engineering, and Industry: 3rd International Conference, Vol. 1, pp. 64-69.

Buonomo, B., Cresci, G., Manca, O., Mesolella, P. and Nardini, S. (2012), "Transient mixed convection in a channel with an open cavity filled with porous media", Journal of Physics: Conference Series Series, Vol. 395 No. 1, doi: 012149.

Buonomo, B., Manca, O., Mesolella, P. and Nardini, S. (2014), "Local Thermal Non-Equilibrium in Mixed Convection in Channels Partially Heated at Uniform Heat Flux Filled With a Porous Medium", ASME 2014 12th Biennial Conference on Engineering Systems Design and Analysis, Copenhagen, pp. V003T012A016-V003T012A016.

Gamrat, G., Favre-Marinet, M. and Le Person, S. (2008), "Numerical study of heat transfer over banks of rods in small Reynolds number cross-flow", International Journal of Heat and Mass Transfer, Vol. 51 No. 3, pp. 853-864.

Khandelwal, M.K. and Bera, P. (2012), "A thermal non-equilibrium perspective on mixed convection in a vertical channel", International Iournal of Thermal Sciences, Vol. 56, pp. 23-34. 
Kuwahara, F., Shirota, M. and Nakayama, A. (2001), "A numerical study of interfacial convective heat transfer coefficient in two-energy equation model for convection in porous media", International Journal of Heat and Mass Transfer, Vol. 44 No. 6, pp. 1153-1159.

Nakayama, A. (1995), PC-Aided Numerical Heat Transfer and Convective Flow, CRC Press, Boca Raton, FL.

Nakayama, A., Kuwahara, F., Umemoto, T. and Hayashi, T. (2002), "Heat and fluid flow within an anisotropic porous medium”, Journal of Heat Transfer, Vol. 124 No. 4, pp. 746-753.

Ozgumus, T. and Mobedi, M. (2015), "Effect of pore to throat size ratio on interfacial heat transfer coefficient of porous media”, Lournal of Heat Transfer, Vol. 137 No. 1, pp. 012602-1-012602-9.

Ozgumus, T., Mobedi, M., Ozkol, U. and Nakayama, A. (2013), "Thermal dispersion in porous mediaa review on the experimental studies for packed beds", Applied Mechanics Reviews, Vol. 65, pp. 031001-1-031001-19.

Oztop, H.F., Al-Salem, K., Varol, Y., Pop, I. and Firat, M. (2012), "Effects of inclination angle on natural convection in an inclined open porous cavity with non-isothermally heated wall", International Journal of Numerical Methods for Heat \& Fluid Flow, Vol. 22 No. 8.

Penha, D.L., Geurts, B., Stolz, S. and Nordlund, M. (2011), "Computing the apparent permeability of an array of staggered square rods using volume-penalization", Computers \& Fluids, Vol. 51 No. 1, pp. 157-173.

Petrasch, J., Meier, F., Friess, H. and Steinfeld, A. (2008), "Tomography based determination of permeability, Dupuit-Forchheimer coefficient, and interfacial heat transfer coefficient in reticulate porous ceramics", International Journal of Heat and Fluid Flow, Vol. 29 No. 1, pp. 315-326.

Rees, D.A.S. (2010), "Microscopic modeling of the two-temperature model for conduction in heterogeneous media", Journal of Porous Media, Vol. 13 No. 2, pp. 125-143.

Rosca, N.C., Rosca, A.V., Grosan, T. and Pop, I. (2014), "Mixed convection boundary layer flow past a vertical flat plate embedded in a non-Darcy porous medium saturated by a nanofluid", International Journal of Numerical Methods for Heat and Fluid Flow, Vol. 24 No. 5, pp. 970-987.

Saeid, N.H. (2004), "Analysis of mixed convection in a vertical porous layer using non-equilibrium model", International Journal of Heat and Mass Transfer, Vol. 47 No. 26, pp. 5619-5627.

Saito, M.B. and de Lemos, M.J. (2005), "A correlation for interfacial heat transfer coefficient for turbulent flow over an array of square rods", ASME Journal of Heat Transfer, Vol. 32 No. 5 , pp. 666-676.

Saito, M.B. and de Lemos, M.J. (2006), "Interfacial heat transfer coefficient for non-equilibrium convective transport in porous media", International Communications in Heat and Mass Transfer, Vol. 128 No. 5, pp. 444-452.

Sheremet, M.A. and Pop, I. (2015), "Free convection in a triangular cavity filled with a porous medium saturated by a nanofluid: Buongiorno's mathematical model", International Iournal of Numerical Methods for Heat \& Fluid Flow, Vol. 25 No. 5.

Teruel, F.E. and Díaz, L. (2013), "Calculation of the interfacial heat transfer coefficient in porous media employing numerical simulations", International Journal of Heat and Mass Transfer, Vol. 60, pp. 406-412.

Wong, K.-C. and Saeid, N.H. (2009), "Numerical study of mixed convection on jet impingement cooling in a horizontal porous layer under local thermal non-equilibrium conditions", International Lournal of Thermal Sciences, Vol. 48 No. 5, pp. 860-870.

Yang, K., You, X., Wang, J. and Vafai, K. (2016), "Analysis of two approaches for an adiabatic boundary condition in porous media", International Journal of Numerical Methods for Heat \& Fluid Flow, Vol. 26 Nos $3 / 4$. 
$\bar{\longrightarrow}$

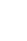

